\title{
Quechua I y Quechua II: En defensa de una distinción establecida
}

\author{
Willem F. H. Adelaar ${ }^{1}$
}

\begin{abstract}
Resumen
La investigación sistemática de las lenguas (o 'dialectos') quechuas, iniciada a partir de los años '60 del siglo XX, creó el fundamento para una división de la familia lingüística quechua en dos subgrupos dialectales, que fueron denominados Quechua I y Quechua II (Torero 1964). El subgrupo Quechua I se concentra en un sector continuo de la sierra central y norteña del Perú, mientras que el subgrupo Quechua II incluye todas las variedades quechuas originarias de otras regiones. En publicaciones recientes (Pearce \& Heggarty 2011; Beresford-Jones \& Heggarty 2012) se ha puesto en cuestión la validez de la bifurcación Quechua I / II y se ha buscado reemplazarla por el modelo de un área continua dialectal, que abarcaría tanto el subgrupo Quechua I como una gran parte de Quechua II. En el presente artículo examinamos los principales argumentos que fueron aducidos en contra o en favor de la dicotomía Quechua I / II. Concluimos que los primeros carecen de fundamento lingüístico empírico y que los últimos están lejos de haber perdido su validez e importancia actual.
\end{abstract}

Palabras clave: Clasificación. Dialectos. Dicotomía. Quechua I / II. Quechua central. Quechua circunferencial. Zona Continua Quechua.

\section{Resumo}

A pesquisa sistemática das línguas (ou 'dialetos') quechuas, iniciada nos anos '60 do século XX, deixou o fundamento para uma divisão da família lingüística quechua em dois subconjuntos dialetais, que foram denominados Quechua I e Quechua II (Torero 1964). O subconjunto Quechua I fica concentrado num setor contínuo da serra central e norte do Peru, enquanto o subconjunto Quechua II inclui todas as variedades quechuas originárias de outras regiões. Em publicações recentes (Pearce \& Heggarty 2011; Beresford-Jones \& Heggarty 2012) a validade da bifurcação Quechua I / II foi questionada e se procurou substituí-la pelo modelo da área contínua dialetal, que abrangeria tanto o subconjunto Quechua I como uma grande parte do Quechua II. No presente artículo examinamos os principais argumentos que foram aduzidos contra ou a favor da dicotomia Quechua I / II. Concluimos que os primeiros carecem de fundamento lingüístico empírico e que os últimos estão longe de ter perdido sua validade e importância atual.

Palavras-chave: Classificação. Dialetos. Dicotomia. Quechua I / II. Quechua central. Quechua circunferencial. Zona Contínua Quechua.

1 Centro de Lingüística de la Universidad de Leiden (LUCL). 


\section{Introducción ${ }^{2}$}

La noción del quechua como una entidad idiomática que engloba varias lenguas se remonta a la época colonial de dominación española. En un vocabulario con la fecha de 1700, Juan de Figueredo refirió al quechua central del Perú como a la lengua chinchaisuyo ('la lengua del norte'), con la implicación que esta fuera una lengua distinta del quechua general, originario del sur del Perú. Aún antes, en 1571, Pedro Pizarro (1986:75) notó diferencias entre el quechua central-peruano de los Xauxas y Guancas (ancestros de la actual población huanca del valle del Mantaro) y el quechua general, comparándolas con las diferencias que separan el castellano del portugués. Sin embargo, la diversidad y, en algunos casos, la profundidad histórica de las subdivisiones geográficas internas del conjunto quechua se hicieron evidentes a mediados del siglo XX, en particular, a raíz de los estudios dialectológicos de Ferrario (1956), Parker (1963) y Torero (1964). En base a una investigación detenida de las características de una multitud de variedades geográficas del quechua (los llamados "dialectos quechuas"), tanto Parker como Torero concluyeron que el conjunto quechua se bifurca en dos ramas primarias. Estos dos grupos, que abarcan la mayor parte, si no la totalidad, de los dialectos quechuas, han sido denominados Quechua I y Quechua II según la nomenclatura original introducida por Torero en 1964 (respectivamente, Quechua B y A en la clasificación de Parker). La rama Quechua I abarca todas las variedades de un bloque dialectal ubicado en el Perú central y central-norteño en los sectores serranos de los departamentos de Ancash, Huánuco, Junín, Lima y Pasco. Este conjunto dialectal formó una zona quechua-hablante continua por lo menos hasta mediados del siglo XX, cuando una parte de las variedades que lo integraban empezó a retroceder ante el castellano. El grupo Quechua II, por su lado, comprende todas las variedades de quechua habladas fuera del área antes definida como perteneciente a Quechua I, tanto al sur como al norte de la misma. También incluye variedades extintas tradicionalmente atribuidas a la costa central del Perú y dialectos localizados en el sector amazónico del Perú, así como todas las variedades de quechua habladas al exterior de este país, o sea, en Argentina, Bolivia, Colombia, Chile y Ecuador.

En varias oportunidades la propuesta de la división del quechua en dos ramas principales llegó a ser cuestionada (Landerman 1991, Mannheim 1991). Sin embargo, en los últimos diez años tal crítica se ha formulado en forma mucho más enfática, con el argumento central que las variedades dialectales del quechua formarían un continuo geográfico con transiciones graduales, en el que no cabe el concepto de una bifurcación inicial dentro de la familia lingüística en cuestión (Heggarty 2005, Pearce \& Heggarty 2011, Beresford-

2 Quedamos profundamente agradecidos a Rodolfo Cerrón-Palomino por su lectura y sus valiosos comentarios del presente artículo. 
Jones \& Heggarty 2012). En realidad, esta representación del quechua como una "cadena de dialectos" (cf. Kaufman 1990) se aplica muy bien a la situación existente dentro de cada uno de los grupos y subgrupos reconocidos dentro de la familia quechua, pero no hace justicia a las diferencias fundamentales que separan sus dos ramas principales (cf. Cerrón-Palomino 2013: 307-308, nota 14) El presente artículo tiene por finalidad la de rectificar la imagen que se puede adquirir de la historia lingüística del quechua en base a críticas precipitadas del llamado modelo "tradicional".

\section{La clasificación de los dialectos quechuas}

En su trabajo pionero de 1964, Torero no sólo propuso la dicotomía del quechua en dos ramas principales, Quechua I y II, sino también una clasificación interna del grupo Quechua II, al que dividió en tres subgrupos: Quechua IIA, IIB y IIC. Los dos subgrupos más numerosos en términos de hablantes, Quechua IIB y Quechua IIC, aunque genealógicamente cercanos, tienen características bien definidas que los distinguen mutuamente. Su distribución geográfica actual también es inequívoca. El subgrupo Quechua IIB se encuentra en el Ecuador, en Colombia, en sectores serranos del noreste del Perú y en el sector amazónico de este país. La variedad extinta de quechua descrita por Domingo de Santo Thomas en 1560 (generalmente asociada con la costa central del Perú), también pertenecía al grupo Quechua IIB, y según toda probabilidad la variante de quechua utilizada en la redacción del llamado manuscrito de Huarochirí, un texto anónimo de alrededor de 1600, proveniente del interior del departamento de Lima (Adelaar 1994). El Quechua IIB se muestra innovador frente al subgrupo Quechua IIC por mostrar una coincidencia de la oclusiva posvelar ${ }^{*} q$ con la oclusiva velar ${ }^{*} k$, el producto de un cambio histórico que se observa en la totalidad del subgrupo IIB y en ninguna otra variedad de quechua hablada en la actualidad. El subgrupo Quechua IIC comprende todas las variedades sureñas del quechua que se hablan en el Perú, al sur de una frontera lingüística y administrativa que separa los departamentos centralperuanos de Junín y Huancavelica, al igual que las variedades habladas en Argentina, Bolivia y Chile.

Dentro del subgrupo Quechua IIC, la variedad quechua de los actuales departamentos peruanos de Ayacucho y Huancavelica, que desborda hacia los departamentos de Apurímac y Arequipa, representa la variedad más conservadora. Esta variedad se encuentra relativamente cercana a un prototipo quechua que se puede reconstruir a partir de los grupos Quechua I y Quechua II unidos y a la vez forma una base de la que las otras variedades del grupo Quechua IIC pueden ser derivadas mediante una serie de cambios fonológicos y morfosintácticos naturales y probables (cf. Adelaar 1995). 
El subgrupo Quechua IIA comprende una serie de variedades locales distribuidas en forma dispersa por partes del Perú central y norteño. La clasificación y la cohesión de este grupo, cuyas características distintivas quedan mal definidas, fueron cuestionadas en varias ocasiones, en primer lugar por su propio proponente (Torero 1974:30). Taylor (1979) prefirió tratar el subgrupo Quechua IIA como una rama independiente al mismo nivel que las demás ramas Quechua I y II ("Quechua III"). El quechua de Pacaraos, ubicado en el alto valle de Chancay en la provincia de Huaral, departamento de Lima, y originalmente asignado al subgrupo IIA (Torero 1964), fue el objeto de una reclasificación tentativa dentro del grupo Quechua I (Parker 1969:191192), o alternativamente como una rama independiente del quechua en su totalidad (Adelaar 1984). Se destaca la posición única del quechua de Pacaraos y su importancia para la reconstrucción del Proto-Quechua. Las demás variedades originalmente clasificadas como Quechua IIA son las variedades quechuas de Cajamarca y de Ferreñafe (departamento de Lambayeque) en el norte del Perú, el grupo dialectal de las comunidades de Lincha, Madeán y Víñac (en la provincia de Yauyos, departamento de Lima), y el dialecto hablado en la comunidad de Laraos de la misma provincia. Mientras que el dialecto de Ferreñafe se asemeja a su vecino cajamarquino, mostrando a la vez una influencia fuerte de Quechua I en su morfología y léxico, las variedades de Cajamarca, de Lincha-Madeán-Víñac, y de Laraos parecen constituir unidades dialectales independientes, que se distinguen por su conservadurismo común y no por innovaciones compartidas. Sus antepasados podrían haberse separado del resto del grupo Quechua II antes de que este se dividiera en los subgrupos IIB y IIC. Las variedades arriba mencionadas que se encuentran en la provincia de Yauyos (Lincha-Madeán-Víñac y Laraos) comparten esta región con una serie de dialectos pertenecientes al grupo Quechua I, y también con el Aimara Central, que consta de las variedades Jaqaru y Kawki (cf. CerrónPalomino 2000). La complejidad lingüística de aquella región ha motivado su cualificación como "microcosmo dialectal" (Taylor 1984).

El Cuadro I da una impresión de la clasificación de los principales dialectos quechuas según la propuesta original de Torero (1964) con las revisiones mencionadas arriba.

\section{Cuadro I}

\begin{tabular}{|l|l|l|}
\hline Quechua I (Perú) & & \\
\hline & Norte. & Ancash, Huánuco, Cajatambo (Lima). \\
\hline
\end{tabular}

3 En una comunicación reciente (en el simposio de REELA, Leipzig, noviembre 2011), César Itier ha cuestionado la existencia de una variedad costeña del quechua que no fuera introducida por los Incas. 


\begin{tabular}{|c|c|c|}
\hline & Sur. & $\begin{array}{l}\text { Norte de Junín-Pasco-Lima (Huaral- } \\
\text { Huaura-Oyón). } \\
\text { Huanca (Jauja, Concepción, Huancayo). } \\
\text { Quechua I de Yauyos (Lima), Chincha } \\
\text { (Ica) y Castrovirreyna (Huancavelica). }\end{array}$ \\
\hline & $\begin{array}{l}\text { Pacaraos } \\
\text { (Huaral, Lima). }\end{array}$ & \\
\hline \multicolumn{3}{|l|}{ Quechua II } \\
\hline & $\begin{array}{l}\text { Cajamarca- } \\
\text { Hualgayoc } \\
\text { (Cajamarca), } \\
\text { Ferreñafe } \\
\text { (Lambayeque). }\end{array}$ & \\
\hline & $\begin{array}{l}\text { Laraos (Yauyos, } \\
\text { Lima). }\end{array}$ & \\
\hline & $\begin{array}{l}\text { Lincha- } \\
\text { Madeán-Viñac } \\
\text { (Yauyos, Lima). }\end{array}$ & \\
\hline & Quechua IIB & $\begin{array}{l}\text { Quechua de Colombia. Quechua del } \\
\text { Ecuador interandino. } \\
\text { Quechua del Oriente ecuatoriano } \\
\text { Quechua de Loreto (Napo y Pastaza, } \\
\text { Perú). } \\
\text { Quechua de Chachapoyas y San Martín } \\
\text { (Perú). } \\
\text { Quechua del manuscrito de Huarochirí } \\
\text { (siglo XVI-XVII; Lima, Perú). } \\
\text { Quechua de la costa central peruana (siglo } \\
\text { XVI; Lima y Chincha, Perú). }\end{array}$ \\
\hline & Quechua IIC & $\begin{array}{l}\text { Quechua de Ayacucho, Huancavelica, } \\
\text { noroeste de Arequipa y oeste de } \\
\text { Apurímac (Perú). } \\
\text { Quechua de Cuzco, Puno, noreste de } \\
\text { Arequipa, este de Apurímac y norte de } \\
\text { Moquegua (Perú). } \\
\text { Quechua del norte de Bolivia. } \\
\text { Quechua del sur y centro de Bolivia (con } \\
\text { noroeste de Argentina y norte de Chile). } \\
\text { Quechua de Santiago del Estero } \\
\text { (Argentina). } \\
\text { Quechua de Catamarca y La Rioja } \\
\text { (Argentina). }\end{array}$ \\
\hline
\end{tabular}


En términos de distribución geográfica es posible afirmar que las lenguas quechuas se dividen primeramente en un grupo central (Quechua I) y un grupo circunferencial (Quechua II). Los principales acontecimientos históricos, que han contribuido a la expansión del quechua más allá del Perú central y sureño, siempre concernieron el grupo Quechua II y no Quechua I. Además, la constitución interna de cada uno de los dos grupos principales del conjunto quechua es diferente. El grupo central (Quechua I) muestra una diversidad bastante grande en el campo fonológico, morfológico y léxico con una transición gradual entre variedades limítrofes. La composición interna de este grupo sugiere un proceso paulatino de diferenciación a través de cambios consecutivos, que parece haber ocupado un largo período de tiempo y que podría haber ocurrido al margen de las empresas bélicas iniciadas por las huestes de Huari (siglos VI-IX) en el Perú central-sureño (región ayacuchana) y por los Incas (siglos XV-XVI) en el sur del Perú (región cuzqueña).

El grupo circunferencial (Quechua II) exhibe una subdivisión más tajante en subgrupos, aunque dentro de cada subgrupo las transiciones dialectales también son graduales. Exhibe las características de una historia movida llena de migraciones, conquistas y ocupaciones de tierras ajenas. Algunas variedades pertenecientes al grupo Quechua II han sufrido cambios fonológicos extremos debido a su trasplantación a tierras previamente ocupadas por hablantes de lenguas muy diferentes, como en el caso del quechua chachapoyano ubicado en el departamento peruano de Amazonas. Sin embargo, la mayoría de las variedades que forman parte de Quechua II se han mantenido relativamente cercanas al prototipo de este grupo. En términos generales, las diferencias internas dentro del grupo Quechua II parecen ser más recientes que las que se encuentran en el grupo Quechua I. Una buena parte de ellas datan de la época del Incanato o de la época colonial.

Aunque la clasificación de la totalidad de los dialectos quechuas según el modelo arbóreo permanece problemático en varios aspectos, la división primaria en dos grupos, Quechua I y Quechua II, fue aceptada en forma general por varias décadas. En particular, la integridad de Quechua I como conjunto más o menos homogéneo raras veces fue cuestionada. En la literatura lingüística relacionada al quechua se suele presentar una lista de criterios, mayormente morfológicos y morfosintácticos, para distinguir los dos grupos. Entre los criterios decisivos que permiten asignar cada variedad particular de quechua a uno u otro de los dos grupos principales, la existencia de distinciones cuantitativas en las vocales y la forma de expresión de la primera persona sujeto y poseedora ocupan un lugar preferencial. Sin embargo, es importante señalar que el establecimiento de una jerarquía en los criterios de diferenciación puede hacer perder de vista el hecho que la identidad de los grupos Quechua I y Quechua II se deriva de toda una serie de características 
mayores y menores, que se encuentran estrechamente interrelacionadas en cada uno de los dos grupos.

\section{Criterios para distinguir entre Quechua I y Quechua II}

Como es de esperar, las diferencias entre los grupos dialectales Quechua I y Quechua II se manifiestan a todos los niveles posibles, en el léxico, en la fonología y en la morfología. Sin embargo, la morfología, por sí sola y en relación con cambios fonológicos ocurridos durante la fase inicial del desarrollo de Quechua I, es la que da a este grupo su carácter propio. Las características morfológicas del grupo Quechua I constan de una mezcla de elementos innovadores y de rasgos conservadores, que se pueden encontrar en forma menos especializada en algunas variedades de Quechua II. En las líneas que siguen compararemos algunos aspectos del léxico, de la fonología y de la morfología de ambos grupos. Desde luego, sólo es posible ofrecer algunos ejemplos de cada sector debido a limitaciones de espacio.

\subsection{El criterio del léxico}

El conjunto dialectal Quechua I comprende un caudal importante de vocabulario propio, que muestra una radiación geográfica limitada y que no sobrepasa los límites de este grupo, salvo ocasionalmente en zonas fronterizas de colonización iniciada por los Incas y por los Españoles, en la que también habrían participado hablantes de Quechua I. Variedades conservadoras de Quechua II, como el quechua de Cajamarca y Ferreñafe y algunas variedades Quechua II de Yauyos, comparten a veces elementos léxicos generalmente atribuidos al grupo Quechua I, lo que sugiere que las fronteras léxicas son fluidas y que estas variedades pueden haber conservado elementos arcaicos o haber asimilado préstamos al nivel local. Algunos elementos léxicos típicamente asociados con Quechua I son nuna 'gente' (en Quechua II runa), $a k s h u$ 'papa (patata)' (en Quechua II, excepto Cajamarca, Laraos y Lincha, papa), haka ( $\left.{ }^{*} s a k a\right)$ 'cobaya' (en Quechua II, excepto todo Yauyos y Ferreñafe, quwi), hunaq 'día' (en Quechua II, excepto Laraos, punchaw < *punĉaw, en quechua huanca y Laraos, muyun), aywa- ir' (en Quechua II ri-, también en quechua de Pacaraos y en quechua huanca bajo la forma li-), qishya'enfermarse' (en Quechua II, excepto Cajamarca y Laraos, unqu-), yarqu'salir' (en Quechua II, excepto Laraos, lluqshi-), urya- 'trabajar' (en Quechua II llamka-), ush(y)a- 'terminar' (en Quechua II tuku-), etc. (cf. Taylor 1994:130-9). Sin embargo, hay que advertir que algunos de estos elementos ni siquiera llegan a cubrir la totalidad del territorio Quechua I (por ejemplo, nuna, akshu, hunaq, y aywa-), mientras que otros lo sobrepasan (por ejemplo, akshu en quechua de Cajamarca, Laraos y Lincha; qishya- en quechua de Cajamarca y Laraos). 
Al revés, el grupo Quechua I no se ha mostrado impermeable a la influencia léxica de Quechua II, debido a la ocupación del territorio de expresión Quechua I por los Incas, los conquistadores y la administración colonial española. En todas estas empresas de inspiración imperial una forma de Quechua II fue siempre el vehículo principal de comunicación impuesto por la fuerza o por la necesidad práctica, lo que no dejó de dejar huellas en el vocabulario de las variedades Quechua I. Por ejemplo, el lexema Quechua II qawa- 'mirar', desconocido en la mayor parte del conjunto Quechua I, se encuentra sin embargo en algunas variedades del departamento de Ancash (Parker y Chávez 1976:137). En el quechua huanca de la región de Huancayo (Quechua I) la forma karan (< * qaran) 'pellejo de cerdo' representa probablemente un préstamo de Quechua II, ya que en la variedad huancaína la oclusiva posvelar /* $\mathrm{q} /$ sufrió un cambio regular a cero en posición inicial de palabra, mientras que el quechua huanca en su totalidad experimentó un cambio fonético de $/{ }^{*} \mathrm{r} / \mathrm{a}$ [1], que de otra manera no permite excepciones (Cerrón-Palomino 1989: 36). Otro caso de préstamo de Quechua II sería la forma yawar 'sangre' que ocurre con /r/ final (y no con [1]) en todo el territorio huanca.

En otros casos más o menos frecuentes el grupo Quechua IIC presenta innovaciones léxicas, mientras que Quechua IIB y Quechua I mantienen formas idénticas. Tal es el caso, por ejemplo, de tamya 'lluvia' que fue reemplazado por para en las variedades sureñas de Quechua IIC. Los pocos ejemplos enumerados arriba sugieren que resulta bastante difícil, después de más de cinco siglos de integración y expansión territoriales, separar el vocabulario de los grupos Quechua I y Quechua II de manera conclusiva.

\subsection{Criterios fonológicos}

La mayoría de las variedades quechuas existentes en la actualidad muestran los resultados de cambios fonológicos ocurridos durante su historia. Los cambios ocurridos en Quechua I no son los mismos que aquellos que se observan en Quechua II. En base a la variedad fonológica que se manifiesta al interior de cada uno de los dos grupos resulta necesario asumir que la mayoría de aquellos cambios haya ocurrido después de la bifurcación que generó los dos grupos. Hay, sin embargo, un acontecimiento histórico de orden fonológico que parece distinguir Quechua I y Quechua II en forma inequívoca. En Quechua I se encuentra una oposición de cantidad vocálica contrastiva, que ha desdoblado el sistema original de tres vocales $(/ \mathrm{a} /, / \mathrm{i} /, / \mathrm{u} /)$ en seis vocales (/a/, /i/, /u/; /a:/, /i:/, /u:/). Se observa una frecuencia mayor de lexemas y formas gramaticales con vocal larga en el sector de las vocales bajas (/a/, /a:/). Con la excepción de algunos dialectos locales de Yauyos y Huancavelica, la oposición de cantidad vocálica es inexistente en Quechua II (aunque puede haber existido en forma marginal en la proto-lengua quechua). 
Una fuente productiva de la vocal larga /a:/ en Quechua I parece haber sido un cambio ocurrido en una fase temprana del desarrollo de este grupo, en el que fue eliminado la semivocal [y] entre dos vocales bajas, es decir, /*aya/ > [a:] (una regla que contó, sin embargo, con un buen número de excepciones, cf. Cerrón-Palomino 1987: 117, y a la que se sobrepusieron nuevas formaciones /aya/ una vez consumido el cambio). La vocal resultante del cambio se mantuvo larga en sílabas abiertas y se abrevió a [a] breve en sílabas trabadas como, por ejemplo, en Quechua I ĉa:shaq 'llegaré' y ĉanki 'llegarás', del verbo ĉa:- (< *ĉya-) 'llegar', formas que se manifiestan, respectivamente, como chayasaq y chayanki en quechua de Ayacucho (Quechua IIC). ${ }^{4}$ Posteriormente, la alternancia de vocales /a/ /a:/ llegó a generalizarse a los sufijos de derivación verbal que terminan en vocal baja, aun cuando no contuvieran originalmente la secuencia /*aya/, de manera que en la actualidad sufijos verbales como - ya(:)$\left({ }^{*}-y k a-\right)$ 'aspecto progresivo' y -ra(:)- ( ${ }^{*}$-raya-) 'continuativo' se comportan de manera idéntica mostrando la misma alternación.

En posición final de palabra se desarrollaron vocales largas con acento final, que tienen por función la de indicar la primera persona del sujeto (con verbos) y del poseedor (con substantivos). ${ }^{5} \mathrm{La}$ indicación de la primera persona por medio de alargamiento vocal en Quechua I se aplica a las tres vocales /a/, /i/, /u/ y se mantiene intacta también cuando no se encuentra en posición final de palabra, aunque en este caso el acento pierde su posición final y no queda necesariamente asociado con la sílaba en que figura la vocal larga. El acento se asigna a la penúltima sílaba (en la mayoría de los dialectos) u a otra sílaba según las reglas acentuales de la variedad en cuestión.

La disponibilidad del contraste de cantidad vocálica en Quechua I ha permitido una proliferación moderada de vocales largas, principalmente en la morfología verbal. En las raíces léxicas las vocales largas siguen siendo poco frecuentes. Sin embargo, las formas verbales pueden contener varias ocurrencias de vocales largas, lo que provoca un contraste marcado con los dialectos Quechua II, en los que la cantidad vocálica es generalmente inexistente.

4 Utilizamos el símbolo $\hat{c}$ para indicar una africada retroflexa. Los símbolos para consonantes palatales $c h, s h, l l, \tilde{n}$ tienen el mismo valor que en castellano peruano. Por lo demás, adoptamos la notación fonética internacional.

5 Las vocales largas en posición final acentuada probablemente tuvieron su origen en un cambio /*v́y\#\#/ > v́:\#\#, ocurrido en una fase temprana del desarrollo de Quechua I (cf. Adelaar 1984). La forma - $v y$ en su calidad de marca de primera persona se encuentra aún en quechua de Pacaraos, y podría haber sido de uso general antes de la separación de las ramas principales del quechua. Tomando en consideración el carácter 'anormal' y, por lo tanto, inestable del acento final en quechua, se puede asumir que en una fase mas temprana del desarrollo de la proto-lengua la terminación de primera persona hubiera sido $/{ }^{*}-\mathrm{yV} /\left({ }^{*}-y a\right.$ en la reconstrucción de Cerrón-Palomino 1987: 139-42) o $/{ }^{*}-\mathrm{Ci} /\left({ }^{*}-h i\right.$ en la reconstrucción de Torero 2002:67). 
Otro rasgo fonológico que se ha mencionado en la literatura pertinente como un posible criterio, que permitiría distinguir entre Quechua I y Quechua II, es la preservación del contraste entre las africadas /ch/ (palatal) y / $\hat{c} /$ (retroflexa) (Itier 2011:65). Sin embargo, este criterio carece de validez ya que algunos dialectos de Quechua II (el cajamarquino, las variedades de Yauyos, y la variedad Quechua IIB de Chachapoyas) mantienen aquella distinción y su presencia anterior también está reflejada en Quechua IIC de Cuzco. Por contraste, algunas variedades de Quechua I han perdido la distinción, mientras que otras han modificado los sonidos respectivos.

\subsection{Criterios morfológicos}

Los principales criterios morfológicos que separan Quechua I y Quechua II se encuentran mencionados con frecuencia en la literatura pertinente (véase, por ejemplo, Torero 1964, 2002: 65-75; Parker 1969, 1971; Cerrón-Palomino 1987:131-152; Adelaar y Muysken 2004:188-190; Pearce y Heggarty 2011:90). En lo que sigue presentamos una selección de criterios, que representa sólo una parte de las indicaciones que favorecen dicha división. ${ }^{6}$

(a) En Quechua I la primera persona con función de sujeto (con verbos) y de poseedor (con substantivos) se indica por medio de alargamiento vocálico, con atracción del acento en posición final de palabra. En la mayoría de las variedades Quechua II se utiliza el sufijo - ni para primera persona sujeto en el indicativo no-futuro (o solo en el presente de indicativo) e $-y$ en el resto del paradigma verbal y como marca poseedora de primera persona de los substantivos. Véanse los siguientes ejemplos:

Quechua I:

muna-: 'yo (lo) quiero', muna-:-man 'yo (lo) puedo querer'

mama-: 'mi madre'

Quechua IIC: ${ }^{7}$

muna-ni 'yo (lo) quiero', muna-y-man 'yo (lo) puedo querer'

mama-y 'mi madre'

[muna- 'querer', -man 'modo potencial'; mama 'madre']

6 Para cada marca gramatical presentada en esta sección sólo indicamos la forma más arcaica en uso, lo que implica que en algunas variedades actuales de quechua se encuentran reflejos o formas fonológicamente modificadas de los elementos mencionados; por ejemplo, en lugar de -shqa también se encuentran las formas -sqa o -sha, según la variación dialectal que queda implícita en esta exposición.

7 Para facilitar la comparación, y en la medida de lo posible, utilizamos raíces verbales cuyas formas son idénticas en los dos conjuntos dialectales principales del quechua. Esto puede dejar la impresión de un parentesco muy cercano, que en realidad no es tan pronunciado. 
Como alternativa para el alargamiento vocálico, varios sub-dialectos de Quechua I manejan una doble marcación del sujeto de primera persona en los verbos, la que implica utilizar el alargamiento vocálico dos veces agregando un elemento conectivo - $n i$-, por ejemplo, en tarpu-pa:ku-:-ni-: 'sembramos' [tarpu- 'sembrar', -pa:ku- 'plural verbal']. Tal forma alternativa podría estar a la base de la marca de sujeto de primera persona no-futuro -ni, que se desarrolló en Quechua II.

(b) En Quechua I la primera persona con función de objeto (directo o indirecto) se indica por medio del sufijo verbal - $m a(:)$-. En casi todos los dialectos Quechua II que marcan objetos de primera persona, el sufijo equivalente es -wa-. Véanse los siguientes ejemplos:

\section{Quechua I:}

muna-ma-nki 'tú me quieres'

Quechua II:

muna-wa-nki 'tú me quieres'

[muna- 'querer', -nki 'sujeto de segunda persona presente y futuro']

Suponemos que en este caso la forma de Quechua II muestra un conservadurismo mayor y que Quechua I - $m a(:)$ - tuvo su origen en la secuencia *-mu-wa-, que sigue siendo usado en Quechua II, como en el siguiente ejemplo del quechua ayacuchano:

kutichi-mu-wa-y 'devuélvamelo'

[kutichi- 'devolver', - $m u$ - 'traslado hacia el hablante', $-y$ 'segunda persona del modo imperativo'].

Así lo sugieren la distribución de - ma(:)- y la imposibilidad de formar secuencias como *-mu-ma(:)- en el Quechua del norte de Junín (véase también Landerman 1991:222-223). ${ }^{8}$

(c) En Quechua I la combinación de una primera persona actora con un objeto de segunda persona se indica por medio del sufijo $-q$, salvo en el quechua huanca y en el quechua de Pacaraos. Estas dos variedades manejan formas especiales que podrían ser el producto de innovaciones locales. Las variedades Quechua IIB y C, en la medida que mantienen la combinación en cuestión, utilizan un sufijo $-(y) k i$, cuya distribución queda limitada a los tiempos de presente y pasado del verbo finito. ${ }^{9}$ Véanse los siguientes ejemplos:

8 Cerrón-Palomino (1987:149) registra la combinación correspondiente -mu-ma(:)en algunas hablas del quechua huanca (Quechua I). A nuestro parecer se trata de una formación nueva, posiblemente debida al contacto con el vecino quechua ayacuchano (Quechua IIC).

9 Esta restricción se debe al hecho que el sufijo - $(y) k i$ también puede indicar un sujeto de segunda persona. La forma $-k i$ sigue a bases que terminan en /i/, por ejemplo, en muna- 
Quechua I:

muna-q 'yo te quiero'

Quechua IIC

muna-yki 'yo te quiero'

El quechua de Cajamarca maneja tanto los sufijos $-q$ (solo en tiempo presente) como - $(y) k i$, y parece captar un proceso histórico de transición desde el uso del primer sufijo hacia el uso del segundo. Concluimos que la forma $-q$ fue la original y que en este respecto las variedades Quechua II se muestran innovadoras en relación con Quechua I (Adelaar 2011).

(d) La manera de indicar la pluralidad de los actantes que participan en el acto expresado por el verbo ofrece un criterio muy importante para distinguir el grupo Quechua I frente a Quechua II. En Quechua I la categoría de plural se indica por medio de sufijos que tienen su lugar al interior del verbo antes de los sufijos que indican tiempo, persona y modo. En general la pluralidad se relaciona con el sujeto, pero en algunos contextos puede referir a un objeto directo o indirecto. Las marcas de plural varían bastante según el dialecto y algunas variedades disponen hasta de tres sufijos que indican pluralidad verbal. Sobre todo en la mitad sur del territorio Quechua I y en quechua de Pacaraos las marcas de pluralidad se interrelacionan con los sufijos de aspecto e inclusive la combinación de aspecto y pluralidad puede ser codificada por sufijos "portmanteau". ${ }^{10}$ Como ilustración, en quechua de Pacaraos el elemento -rka:ĉa(:)- indica la combinación de la pluralidad verbal con el aspecto progresivo y en quechua del Norte de Junín el elemento -ra:ri-indica la combinación de la pluralidad verbal con el aspecto perfectivo. Véanse los siguientes ejemplos:

Quechua de Pacaraos:

rima-rka:ĉa-n 'ellos están hablando'

Quechua del Norte de Junín:

rima-ra:ri-n 'ellos han hablado'

[rima- 'hablar', - $n$ 'sujeto de tercera persona tiempo presente]

Por contraste, en Quechua IIB/C se ha desarrollado un sistema muy característico de marcar el plural en una posición periférica al verbo, detrás de los sufijos de tiempo, persona y modo (con apenas una excepción en lo que concierne al modo). En estas variedades los sufijos de pluralidad ( $k u$ para primera y tercera persona y -chik/-chis para la segunda) se interrelacionan con

pti-ki 'si/cuando/porque (lo) quieres'.

10 Hay que observar que la interconexión de aspecto y plural también se encuentra en aimara, pero no en quechua del sur. 
las marcas de persona hasta realizarse una fusión parcial de las dos categorías en algunas variedades quechuas de Argentina y Bolivia (Adelaar 1995). Los sufijos de pluralidad de Quechua IIB/C también se aplican para pluralizar el poseedor de un substantivo, mientras que en Quechua I normalmente no se marca la pluralidad del poseedor. Las variedades quechuas del norte del Perú (Cajamarca, Ferreñafe, Chachapoyas) igualmente utilizan sufijos externos para indicar la pluralidad, pero estos son de formación reciente y no tienen ninguna relación formal con las marcas de plural del Quechua II sureño. Otros miembros del antiguo grupo Quechua IIA parecen desconocer la marcación de pluralidad en el verbo y en la posesión del substantivo (Taylor 1984:128). Todo esto indica que los sistemas de marcación de pluralidad se desarrollaron independientemente en los dos grupos principales del quechua.

(e) En Quechua I la marca de subordinación verbal en el caso que los sujetos del verbo subordinado y del superordinado son idénticos ('switchreference same subjects') es $-r$, asimismo en Ferreñafe y Laraos (Quechua II). Las otras variedades Quechua II y el quechua de Pacaraos manejan la forma -shpa (separable en -sh + -pa en esta última variedad). El quechua de Huallaga (Huánuco, Quechua I) maneja las dos formas (Weber 1989:298-299).

(f) Pearce y Heggarty (2011:90) mencionan el caso del sufijo de aspecto progresivo $-y k a(:)-$ de Quechua I y quechua de Pacaraos y su equivalente -chka- de Quechua IIC y Laraos. Es posible que el uso de -yka(:)- sea una característica de Quechua I. Hay que observar sin embargo que el quechua huanca (Quechua I) maneja las dos formas con una diferencia de significado, 'acción anticipada' en el caso de -chka(:)- (Cerrón-Palomino 1976:211-213), y que en Quechua II de Cajamarca coexisten las dos formas - yka- y -ĉka-con la misma diferencia de significado (Quesada 1976:133-134). Además, algunos dialectos de Quechua IIB manejan reflejos de $-y k a-$ (Chachapoyas y San Martín) y otros desconocen ambas formas (quechua de Ecuador). Por eso, la alternativa $-y k a(:)-/$-chka- $\sim-\hat{c} k a-$ no parece ser un criterio confiable para distinguir entre Quechua I y Quechua II (cf. Adelaar 2012:210).

(g) Casi todas las variedades de Quechua I manejan una forma verbal que termina en el sufijo - ñaq o -naq. Este sufijo ocupa funciones algo distintas según el dialecto, pero en la parte sur de Quechua I forma parte del sistema de tiempos verbales y ocurre acompañado por el verbo auxiliar $k a$ - 'ser'. Su única función en quechua del Norte de Junín y en quechua de Pacaraos es esta de indicar una revelación o un evento sorpresivo. El sufijo -ñaq o -naq es inexistente en Quechua IIB/C, donde la función de revelación y sorpresivo es generalmente expresada por la marca de nominalización -shqa, de uso general en todo el quechua, en combinación con el verbo auxiliar 'ser'. La introducción de -ñaq / -naq debe ser considerada como una innovación de Quechua I. 
(h) El grupo Quechua I tiene un juego completo de cuatro sufijos verbales que indican dirección, -rpu- 'hacia abajo', -rku- 'hacia arriba', $-y k u$ - 'hacia adentro', -rqu- 'hacia afuera'. Todos son productivos, pero $-y k u-\mathrm{y}-r q u$ mantienen su función original sólo en formas fosilizadas. En Quechua IIB/C los sufijos $-r p u-\mathrm{y}-r k u$ - son inexistentes, salvo en algunas formas fosilizadas; del mismo modo que en Quechua I, los sufijos - yku- y -rqu- mantienen su función original sólo en formas fosilizadas.

(i) De los sufijos de caso nominal sólo unos cuantos coinciden en Quechua I y Quechua II, notablemente, el acusativo - $(k) t a$, el alativo -man, el genitivo - pa y el benefactivo -paq. Las marcas de caso locativo, ablativo y comparativo son completamente distintas. En Quechua I y quechua de Pacaraos el sufijo de caso locativo es - $\hat{c} a w$; su equivalente en Quechua II es generalmente -pi. El ablativo en Quechua I y quechua de Pacaraos se expresa por -piq, -piqta o -pita; su equivalente Quechua II (con excepción de Laraos) es -manta. Quechua I, quechua de Pacaraos y quechua de Ferreñafe (Quechua II) manejan un sufijo -naw para el caso comparativo. Algunos dialectos también conocen la forma -yupay. En Quechua II B/C se utiliza generalmente una forma libre hina 'como' para marcar la comparación.

\section{Los argumentos en contra de la distinción Quechua I / Quechua II y su viabilidad}

Los argumentos aducidos contra la hipótesis de una bifurcación del quechua en dos grupos Quechua I y Quechua II son de varios tipos. Resulta difícil clasificar la totalidad de las variedades quechuas en la estructura binaria propuesta según un criterio particular decisivo. La búsqueda de tal criterio tiene su valor como ejercicio académico pero deja de lado el hecho que la identidad de cada grupo se basa en un haz de criterios y no en uno solo.

En su estudio de los dialectos quechuas, Landerman (1991) observa que la clasificación del quechua en grupos y subgrupos de variedades es problemática, ya que los dialectos del controvertido subgrupo Quechua IIA no muestran la unidad esperada, es decir, no muestran innovaciones compartidas. Esto significa que puede ser necesario reformular la clasificación interna del quechua por medio de la agregación de subgrupos adicionales. Además, Landerman señala la escasez de innovaciones propias del grupo Quechua II, la que puede ocasionar dudas respecto a su coherencia inicial. Sin embargo, este autor no abandona el modelo arbóreo y no niega la existencia de Quechua I como grupo delimitado reconocible dentro del panorama de los dialectos quechuas. La observación de Beresford-Jones \& Heggarty (2012:73) que según Landerman la división Quechua I / Quechua II sería “insostenible" ('untenable') no hace justicia a la realidad. De nuestra parte queremos subrayar la coherencia interna del grupo Quechua I y la profundidad histórica que la caracteriza. 
En el campo fonológico, Mannheim (1991) señala una coincidencia en el comportamiento de una de las dos sibilantes reconstruidas para el quechua sureño (Quechua IIC) y una sibilante que todavía se encuentra en uso en el quechua huanca, el representante más meridional del grupo Quechua I, cuyo territorio colinda con el quechua ayacuchano (Quechua IIC). Esta sibilante se manifiesta como sibilante prepalatal [̌̌] en la mayor parte de Quechua I y Quechua IIB; como "sibilante apical ligeramente retroflexa" [ $\hat{\mathrm{s}}]$ en el quechua huanca (Cerrón-Palomino 1987:162-163) y opcionalmente como sibilante ápico-alveolar [ş] en Porcón, Cajamarca (Quesada: 1976: 41-42). Esta sibilante coincide con [s] alveo-dental (dorsal) en el quechua sureño (Quechua IIC). Sin embargo, durante la época colonial el quechua sureño aún distinguía dos sibilantes, representadas como $<\mathrm{s}, \mathrm{ss}>\mathrm{y}<\mathrm{c}, \mathrm{c}, \mathrm{z}>$. La sibilante representada por $<$ s, ss $>$ sería una sibilante apical parecida a la sibilante arriba mencionada del quechua huanca. En base a estos hechos Mannheim (1991:175-176) concluye que el quechua huanca se encuentra más cercano al quechua sureño que el resto de Quechua I y que, por lo tanto, la bifurcación inicial de los dialectos quechuas en dos grupos principales necesita ser reconsiderada. En contra de esta conclusión caben las objeciones siguientes: (a) la descripción fonética de la sibilante apical del quechua sureño permanece especulativa, ya que no sobrevivió en las variedades actuales de este conjunto dialectal; (b) aún cuando dichas sibilantes del huanca y del quechua sureño hubieran sido fonéticamente idénticas, no se puede excluir una extensión por difusión desde el quechua sureño hacia el quechua huanca, con el que se encuentra limítrofe; y (c) la objeción principal: la presencia de una sola coincidencia fonética superficial y difusible cae en la nada frente a la multitud de elementos distintivos que separan el conjunto Quechua I de las demás variedades del quechua y del quechua sureño en particular. Para remate se puede observar que tanto el quechua huanca, como el quechua de Cajamarca conservan una africada retroflexa, que puede haber influido en la realización de una sibilante que originalmente tal vez no lo era.

En su artículo reciente, que parece tener por meta la de acabar con el llamado "modelo tradicional" de la bifurcación histórica del conjunto quechua en dos grupos primarios, Pearce \& Heggarty (2011) examinan las dimensiones socio-históricas de la línea divisora que separa los territorios de habla Quechua I y Quechua II. Esta línea, también conocida como "la línea de Ñahuinpuquio", coincide globalmente con el límite administrativo que separa los departamentos de Junín y Huancavelica en los Andes centrales del Perú. Los autores asumen una expansión histórica del quechua ayacuchano (Quechua II) hacia el noroeste debido a la explotación de las minas de mercurio en Huancavelica y la concentración de quechua-hablantes de origen no-nativo en aquella región durante la época colonial. Por causa del éxito del habla quechua asociada con la región de Ayacucho y Huancavelica, esta variedad de Quechua 
IIC se habría superpuesto a otras variedades de quechua que otrora hubieran formado una transición gradual entre el grupo dialectal huanca (Quechua I) y el ayacuchano (Quechua IIC), dejando como huella del proceso una "frontera lingüística” bien marcada.

De hecho, la creación de una frontera lingüística como resultado de la actividad minera colonial resulta plausible. Sin embargo, esto no quiere decir que los dialectos desplazados hayan configurado una transición gradual. Es igualmente posible asumir la existencia previa de otra frontera lingüística más hacia el sureste y tal vez menos abrupta, pero de igual modo real. Además existe la posibilidad que los territorios de Quechua I y Quechua II originalmente fueran separados por grupos de hablantes de otras lenguas. En Pearce \& Heggarty (2011), asimismo en Beresford-Jones \& Heggarty (2012), la línea de Nahuinpuquio se plantea como una cesura de tipo histórico secundario, una especie de irregularidad en la llamada "Zona Continua Quechua”. El término "Zona Continua" fue introducido por Torero (1964) con el sentido puramente geográfico de un conjunto de dialectos quechuas hablados en un territorio ininterrumpido. Sin embargo, en la conceptualización de los autores británicos este término parece haber llegado a referirse a un continuo dialectal en el sentido lingüístico de la expresión.

Como para restar importancia a la divisoria lingüística de Nahuinpuquio, Pearce \& Heggarty procuran calificar a la región multidialectal de Yauyos como “sub-continuo" y como un "eslabón perdido" ('missing link'), que hubiera perdurado como muestra del carácter gradual de la transición entre Quechua I y Quechua II. Se hace la comparación con el conocido "abanico renano", sector en el que varias isoglosas fonológicas interrelacionadas, que forman la división entre el bajo alemán y el alemán central, se separan en un lugar determinado para volver a unirse en otro. Sin embargo, esta comparación no se aplica de ningún modo a la región de Yauyos, en la que las numerosas variedades locales lograron conservar su carácter respectivo de Quechua I o Quechua II, pese a la inevitable interacción entre dialectos comunitarios de pocos hablantes que comparten un espacio geográfico reducido. Por lo menos, tal es la realidad que se trasluce de la información detallada sobre Yauyos presentada en los trabajos de Taylor $(1984,1994)$.

En su afán de poner en cuestión la coherencia y viabilidad del grupo Quechua I, Pearce \& Heggarty (2011: 96-8) buscan inventariar las propiedades idiosincráticas del conjunto dialectal huanca, localizado en el sureste del departamento de Junín, las que permitirían calificar este conjunto dialectal como una zona de transición entre los grupos Quechua I y Quechua II y no como una parte inherente del primero. Entre aquellas propiedades mencionan el uso de /1/, producto de la lateralización del antiguo fonema / $\mathrm{r} /$, una característica de la zona, y la existencia en una sub-zona de la región huanca 
de una forma especial ya?a $\left(<{ }^{*} y a q a\right)$ para el pronombre personal de primera persona (en lugar de la forma general ñuqa). Evidentemente, se trata de innovaciones ocurridas en (una parte de) la región huanca, que no favorecen su relacionamiento ni con Quechua I ni con Quechua II.

Por otro lado, los autores destacan la existencia en huanca de un artículo definido sufijado, que sólo se encuentra en este conjunto dialectal. Esta marca de definición puede asumir varias formas según el sub-dialecto y su posición en la palabra: -kaq, -ka?-, -ka:-, -ka, -?- o -(V):- (cantidad vocálica). Véase el siguiente ejemplo:

Quechua huanca de Chongos Bajo (Huancayo):

wamla-ka 'la muchacha', wamla-:-ta 'a la muchacha'

[wamla 'muchacha', -ta 'caso acusativo']

Sin embargo, a pesar de ser único para la totalidad del quechua el aporte semántico de esta marca de definición huanca, su forma se deriva fácilmente del elemento individualizador $k a-q$ (nominalización agentiva del verbo $k a-$ 'ser'), utilizado con frecuencia en los dialectos Quechua I situados hacia el norte del conjunto huanca para derivar frases substantivas, lo que traiciona el origen reciente e innovador del artículo definido huanca. Véase el siguiente ejemplo:

Quechua del Norte de Junín:

ali ka-q 'el bueno', 'lo bueno'

[ali 'bueno', $k a-$ 'ser', $-q$ 'nominalización agentiva'].

Finalmente, Pearce \& Heggarty (2011:96) parecen atribuir un carácter arbitrario a las vocales largas encontradas en huanca, "mas frecuentes que en otras regiones quechuas, en lugares muy inesperados y con significados totalmente diferentes" (traducción nuestra). Esta observación, bastante ligera en su fundamento empírico, parece ignorar el hecho que las vocales largas del huanca son básicamente las mismas que en los demás dialectos de la mitad sur del territorio Quechua I, con la diferencia que en una parte de la región huanca (Cerrón-Palomino 1987:160-161; 1989:68-71) la secuencia -VPC- (< ${ }^{*}-V q C$-) sufrió un cambio a $-V: C$-, en el contexto de una tendencia general al desgaste de [?] ( $\left.{ }^{*} q\right)$, lo que ocasionó un aumento de los casos de palabras y formas gramaticales con vocal larga y al mismo tiempo un reajuste de algunas reglas de alargamiento vocálico pre-existentes (cf. Adelaar 1984:30-36). En realidad, las distintas variedades de Quechua I, y en particular aquellas de la mitad sur de este conjunto dialectal, muestran un estrecho parentesco en el uso de la cantidad vocálica y las reglas morfo-fonológicas que la conciernen. Las correspondencias detalladas e idiosincráticas en este dominio, que unen al quechua huanca con las variedades quechuas habladas en el noroeste de Junín, 
Pasco y Lima, comprueban de manera convincente la unidad histórica de todo el sector meridional del grupo Quechua I y, por extensión, de la totalidad de este conjunto dialectal caracterizado por una transición gradual de sus variedades. Por contrario, es necesario enfatizar que el quechua ayacuchano, al igual que todo el Quechua IIB/C, desconoce las vocales largas y ni siquiera tiene huellas de su posible existencia anterior.

Para completar su argumentación con relación a la supuesta posición transicional del quechua huanca, Pearce \& Heggarty (2011:97-98) mencionan una serie de coincidencias que parecen unir el sector meridional de este conjunto dialectal con el Quechua IIC de Ayacucho y Huancavelica. Se trata por lo general de elementos morfo-sintácticos superficiales, por ejemplo, el uso de -nki en lugar de - $y k i$ para indicar un sujeto verbal de segunda persona en el tiempo pasado, el uso del sufijo de caso -man en lugar de -(k)ta para marcar un objeto indirecto en los substantivos, así como el uso del sufijo diminutivo - cha. Todos estos elementos pueden ser interpretados sin mayor problema como el producto de una difusión horizontal desde el Quechua IIC hacia la zona fronteriza del quechua huanca. ${ }^{11}$

\section{Conclusiones}

En las páginas anteriores solo hemos tocado a algunos aspectos superficiales de la realidad compleja en la que se basa la identidad lingüística e histórica del grupo Quechua I. Un estudio más detallado y detenido de los mecanismos fonológicos y morfo-sintácticos compartidos por las distintas variedades que lo componen podría revelar una visión más completa de la unidad filogenética de este conjunto dialectal y permitir una mejor apreciación de la antigüedad de los procesos que subyacen sus relaciones internas. Al mismo tiempo, los criterios que fueron avanzados en favor de la dicotomía Quechua I / Quechua II en la literatura fundadora de la dialectología quechua siguen manteniendo validez en su gran mayoría. Por contraste, ni uno de los argumentos de índole lingüística aducidos en la literatura de las últimas dos décadas en contra de la división original del quechua en dos ramas primarias se muestra resistente al escrutinio científico. Por lo tanto, no resulta justificado rechazar la existencia de una dicotomía histórica Quechua I / II basándose en estas críticas.

Aunque la clasificación "tradicional" en dos conjuntos dialectales que juntos constituyen la familia lingüística quechua sigue en pie, el carácter asimétrico de la relación entre las dos ramas merece mayor atención. La diversidad interna

11 Pearce \& Heggarty (2011:97) también mencionan la selección alternativa de las marcas de acusativo - ta y - kta para indicar el objeto directo. El sufijo - kta solamente se encuentra en quechua huanca, en quechua de Pacaraos y en variedades de Quechua IIB/C registradas durante los siglos XVI-XVIII. Se trata de una forma arcaica, que solo fue conservada en las dos variedades arriba mencionadas. 
propia de cada grupo tiene un carácter diferente, lo que podría ser relacionado con factores de orden cronológico. La diversidad interna del grupo Quechua I refleja una secuencia de cambios innovadores de tipo gradual y de extensión local, que podrían haber ocupado un período de tiempo considerable y que sin duda habrían empezado mucho antes de la unificación incaica, posiblemente al margen del desarrollo del estado Huari (siglos VI-IX). En cambio, el grupo Quechua II, aparentemente menos innovador en su fase inicial, refleja una historia de diversificación reciente con rupturas abruptas, migraciones de larga distancia y desplazamientos lingüísticos. Aunque la proto-lengua del conjunto Quechua II probablemente también remonta al período Huari una gran parte de su diferenciación interna ocurrió durante la época de expansión incaica y durante la colonia. En un análisis de la diversidad geográfica del quechua resulta esencial reconocer las diferencias de antigüedad que caracterizan las relaciones interdialectales en el quechua actual, tomando en cuenta al mismo tiempo los movimientos niveladores que han afectado a esta familia lingüística durante su historia y el contacto idiomático constante entre sus múltiples variedades locales.

\section{Referencias}

Adelaar, Willem F.H. 1984. 'Grammatical vowel length and the classification of Quechua dialects.' International fournal of American Linguistics 50:25-47.

Adelaar, Willem F.H. 1994. 'La procedencia dialectal del manuscrito de Huarochirí en base a sus características lingüísticas'. Revista Andina, 12(1):137-154. Cuzco: Centro de Estudios Regionales Andinos "Bartolomé de Las Casas".

Adelaar, Willem F.H. 1995. 'Raíces lingüísticas del quechua de Santiago del Estero.' En: Ana Fernández y Pedro Viegas (editores), Actas II fornadas de Lingüística Aborigen (Buenos Aires 1994), pp. 25-50. Buenos Aires: Facultad de Filosofía y Letras, Instituto de Lingüística.

Adelaar, Willem F.H. 2011. 'Reconstruyendo el paradigma verbal quechua: El caso de la transición de primera a segunda persona.' En: Willem Adelaar, Pilar Valenzuela y Roberto Zariquiey (editores), Estudios sobre lenguas andinas y amazónicas. Homenaje a Rodolfo Cerrón-Palomino, pp. 21-31. Lima: Fondo Editorial de la Pontificia Universidad Católica del Perú.

Adelaar, Willem F.H. 2012. 'Cajamarca Quechua and the expansion of the Huari state.' En: David Beresford-Jones y Paul Heggarty (editores), Archaeology and Language in the Andes, pp. 197-217. Proceedings of the British Academy. Oxford: Oxford University Press.

Adelaar, Willem F.H., y Pieter C. Muysken. 2004. The Languages of the Andes. Cambridge, Reino Unido: Cambridge University Press.

Beresford-Jones, David, y Paul Heggarty. 2012. 'Broadening Our Horizons: Towards an Interdisciplinary Prehistory of the Andes.' En: David Beresford-Jones y Paul 
Heggarty (editores), Archaeology and Language in the Andes, pp. 57-84. Proceedings of the British Academy. Oxford: Oxford University Press.

Cerrón-Palomino, Rodolfo M. 1976. Gramática quechua funín-Huanca. Lima: Ministerio de Educación e Instituto de Estudios Peruanos.

Cerrón-Palomino, Rodolfo M. 1987. Lingüística quechua. Cuzco: Centro de Estudios Regionales Andinos "Bartolomé de Las Casas".

Cerrón-Palomino, Rodolfo M. 1989. Lengua y sociedad en el valle del Mantaro. Lima: Instituto de Estudios Peruanos.

Cerrón-Palomino, Rodolfo M. 2000. Lingüística aimara. Lima: Centro de Estudios Regionales Andinos "Bartolomé de Las Casas".

Cerrón-Palomino, Rodolfo M. 2013. Las lenguas de los incas: el puquina, el aimara y el quechua. Frankfurt am Main: Peter Lang.

Ferrario, Benigno. 1956. 'La dialettologia ed i problema interni della Runa-Simi (vulgo Quéchua)'. Orbis 5:131-40. Lovaina.

Figueredo, Juan de. 1964 [1700]. 'Vocabulario de la lengua chinchaisuyo y algunos modos mas usados en dicha lengua.' En: Luis Pardo y Carlos Galimberti (editores), Diego de Torres Rubio: Arte de la lengua quichua, pp. 112-20. Cuzco: H.G. Rozas.

Heggarty, Paul. 2005. 'Enigmas en el origen de las lenguas andinas: aplicando nuevas técnicas a las incógnitas por resolver.' Revista Andina 40:9-80. Cuzco: Centro de Estudios Regionales Andinos "Bartolomé de Las Casas".

Itier, César. 2011. 'What was the Lengua General of Colonial Peru?' En: Paul Heggarty y Adrian J. Pearce (editores), History and Language in the Andes, pp. 63-85. Nueva York: Palgrave Macmillan.

Kaufman, Terrence S. 1990. 'Language history in South America: What we know and how to know more.' En: Doris Payne (editora), Amazonian Linguistics: Studies in Lowland South American Languages, pp. 13-73. Austin: University of Texas Press.

Landerman, Peter N. 1991. Quechua Dialects and their Classification. Tesis de doctorado, UCLA. Los Angeles.

Mannheim, Bruce. 1991. The Language of the Inka since the European Invasion. Austin: University of Texas Press.

Parker, Gary J. 1963. 'La clasificación genética de los dialectos quechuas.' Revista del Museo Nacional 32:241-52. Lima.

Parker, Gary J. 1969. 'Comparative Quechua phonology and grammar IV: The evolution of Quechua A.' Working Papers in Linguistics 1(9):149-204. Honolulu: Department of Linguistics, University of Hawaii.

Parker, Gary J. 1971. 'Comparative Quechua phonology and grammar IV: The evolution of Quechua B.' Working Papers in Linguistics 3(3):45-109. Honolulu: Department of Linguistics, University of Hawaii.

Parker, Gary J., y Amancio Chávez. 1976. Diccionario quechua Ancash-Huailas. Lima: Ministerio de Educación e Instituto de Estudios Peruanos. 
Pearce, Adrian J., y Paul Heggarty (2011) 'Mining the Data on the HuancayoHuancavelica Quechua Frontier'. En: Paul Heggarty y Adrian Pearce (editores) History and Language in the Andes, pp. 87-109. Nueva York: Palgrave Macmillan.

Pizarro, Pedro. [1571] 1986. Relación del descubrimiento y de la conquista de los reinos del Perú. Lima: Fondo Editorial de la Pontificia Universidad Católica del Perú.

Quesada Castillo, Félix. 1976. Gramática quechua Cajamarca-Cañaris. Lima: Ministerio de Educación e Instituto de Estudios Peruanos.

Santo Thomas, Domingo de. [1560] 1994. Grammatica o arte de la lengua general de los Indios de los Reynos del Perú. Edición facsimilar. Estudio y transliteración de Rodolfo Cerrón-Palomino. Madrid: Ediciones de Cultura Hispánica.

Taylor, Gerald. 1979. 'Morphologie comparée du verbe quechua: L'expression de l'actance, Première partie: Le Sujet'. En: Cathérine Paris (editora), Relations prédicatactant(s) dans des langues de types divers II, pp. 171-86. LACITO-documents. Paris: SELAF.

Taylor, Gerald. 1984. Yauyos, un microcosmo dialectal quechua, Revista Andina 2(1):121-46. Cuzco: Centro de Estudios Regionales Andinos "Bartolomé de Las Casas".

Taylor, Gerald. 1994. Estudios de dialectología quechua (Chachapoyas, Ferreñafe, Yauyos). Lima: Universidad Nacional de Educación.

Torero, Fernández de Córdova, Alfredo A. 1964. 'Los dialectos quechuas.' Anales Científicos de la Universidad Agraria 2(4):446-78. La Molina (Lima).

Torero Fernández de Córdova, Alfredo A. 1974. El quechua y la historia social andina. Lima: Universidad Ricardo Palma.

Torero Fernández de Córdova, Alfredo A. 2002. Idiomas de los Andes. Lingüística e historia. Lima: Instituto Francés de Estudios Andinos y Editorial Horizonte.

Weber, David J. 1989. A Grammar of Huallaga (Huánuco) Quechua. University of California Publications in Linguistics, volumen 112. Berkeley y Los Angeles: University of California Press.

Data recebimento: $10 / 05 / 2013$

Data aceite: $27 / 05 / 2013$ 